\title{
Report
}

\section{Novel Discovery of the Relationship of Clinical Phenotype of Coronary Heart Disease with Retinol Binding Protein 4, Lipoprotein-Related Phospholipase A2}

\author{
Liu-qiang Lv ${ }^{1}$, Yang-zhang Tang ${ }^{1}$, Shi-qiang Wang ${ }^{1}$, Gui Ren ${ }^{2}$, Yu-Chen Lo ${ }^{3}$, Hiroshi Honda ${ }^{2}$, \\ Edward J. Parish ${ }^{4}$ \\ ${ }^{1}$ Department of Cardiovascular Medicine, Huaibei People's Hospital, Huaibei, China \\ ${ }^{2}$ Department of Bioengineering, Northwestern Polytechnic University, Fremont, USA \\ ${ }^{3}$ Department of Bioengineering, Stanford University, Palo Alto, USA \\ ${ }^{4}$ Department of Chemistry, Auburn University, Auburn, USA
}

\section{Email address:}

chrishonda@yahoo.com (H. Honda)

\section{To cite this article:}

Liu-qiang Lv, Yang-zhang Tang, Shi-qiang Wang, Gui Ren, Yu-Chen Lo, Hiroshi Honda, Edward J. Parish. Novel Discovery of the Relationship of Clinical Phenotype of Coronary Heart Disease with Retinol Binding Protein 4, Lipoprotein-Related Phospholipase A2. Clinical Medicine Research. Vol. 6, No. 4, 2017, pp. 131-134. doi: 10.11648/j.cmr.20170604.13

Received: May 2, 2017; Accepted: May 18, 2017; Published: June 29, 2017

\begin{abstract}
To investigate the correlation between retinol binding protein 4 (RBP4), lipoprotein associated phospholipase A2 (LP-PLA2), we studied the clinical phenotype of coronary heart disease (CHD) and the severity of coronary artery disease. Our studies showed that the changes of retinol binding protein 4, LP-PLA2 serum concentration has a relation with the severity of coronary artery disease progression, but no clear relationship with the degree of coronary artery lesion and count. Furthermore, LP-PLA2 concentration increases with the increase of the severity of coronary artery disease and disease occurrences.
\end{abstract}

Keywords: Retinol Binding Protein 4, Lipoprotein Associated Phospholipase A2, Coronary Heart Disease, Coronary Artery Lesion Degree

\section{Introduction}

Coronary heart disease is one of the common diseases in cardiology. Many risk factors and chronic inflammatory reaction can lead to the formation of coronary heart disease. One of the common risk factors is dyslipidemia. Lipid deposition in the vascular wall, causing atherosclerosis and plaque formation lumen narrowing, results in loss of oxygen and oxygen consumption out of balance. Likewise, plaque rupture can lead to a series of coronary events, in which the inflammatory reaction in the arteriosclerosis process plays a certain role in triggering. Consequently, early detection of chronic inflammatory reactions offers a potential strategy for the cardiovascular disease prevention.

Monitoring serum metabolites is one of the most effective way for detecting heart diseases. Some commonly used biomarkers for the heart disease prediction including
C-reactive protein, apolipoproteins, lipoprotein-associated phospholipase A2, and B-type natriuretic peptide. Here, we reported two additional metabolites RBP4 and LP-PLA2 as novel biomarkers (Figure 1) for cardiovascular condition. RBP4 is a novel adipocyte-derived cytokine that mediates chronic inflammatory response and promotes insulin resistance (IR), leading to further metabolic disorders of glucose and lipid [1]. These metabolic disorders are the major risk factors for coronary heart disease. Similarly, LP-PLA2 is a newly discovered inflammatory factor, and has shown an important role in the promotion of atherosclerosis formation and development process [2]. In this report, the concentrations of RBP4 and LP-PLA2 in different populations were evaluated, and the values of RBP4 and LP-PLA2 in predicting the different types of coronary artery disease and the degree of coronary artery disease were assessed. 
a.

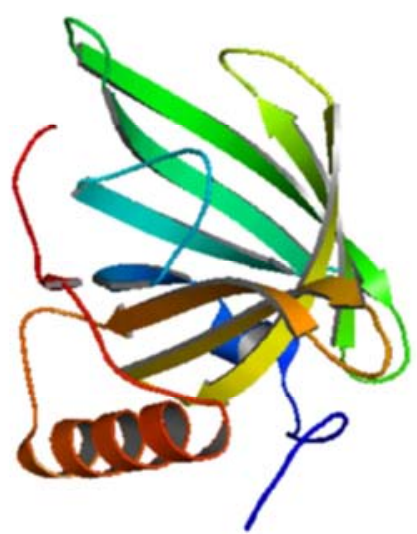

b.

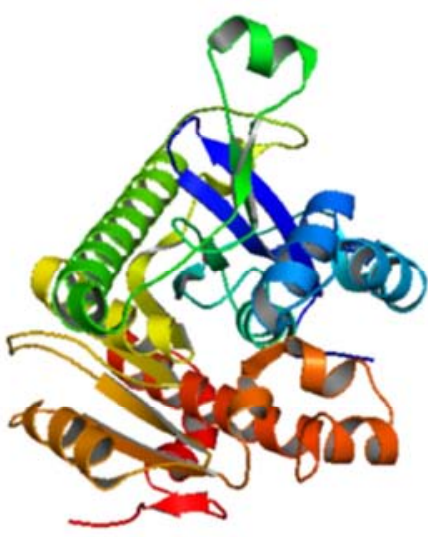

Figure 1. The identification two serum metabolites as novel biomarkers for Coronary heart disease Detection: (a) RBP4 (PDB: 4O9S). (b) LP-PLA2 (PDB: $5 L P 1)$.

\section{Materials and Methods}

\subsection{General Information}

From September 2013 to March 2014, 160 patients with coronary heart disease were selected from our hospital, including 102 males and 58 females with an average age of $(65.37 \pm 7.52)$ years and all the selected patients undergone coronary angiography. The selection criteria are based on the 2012 Chinese Medical Association coronary atherosclerotic heart disease diagnosis and classification criteria [3]. Based on the results of coronary angiography, 112 patients were divided into two groups: the coronary artery disease group (treatment group), and the control group of 48 cases. Patients with the following conditions are excluded including primary and secondary cardiomyopathy, rheumatic heart disease, senile valve disease, infectious diseases, severe hepatitis, severe renal insufficiency and so on. There was no significant difference between the two groups in age, gender differences, and duration of disease, history of smoking, blood pressure and body mass ratio $(\mathrm{P}>0.05)$.

\subsection{Coronary Angiography and Coronary Heart Disease Diagnostic Criteria and the Determination of the Number of Coronary Artery Disease}

Coronary angiography method Judkins method, the radial artery approach, in the DSA room (catheter room) coronary angiography, there are at least two qualified physicians in our division of our hospital to complete the acquisition of orthotopic, liver, right shoulder position, head bit, left shoulder position, spider bit irradiation image. Acute myocardial infarction, unstable angina, stable angina diagnosis consistent with the guideline of coronary atherosclerotic heart disease diagnosis and classification criteria [3]. The criterias to determine the number of lesions are: (1) single branch disease: refers to the anterior descending artery, circumflex artery, right coronary artery in any one involvement, diameter stenosis greater than $50 \%$; (2) double-vessel disease: refers to the anterior descending artery, circumflex artery, right coronary artery in the Diameter stenosis greater than 50\%; (3) three lesions: anterior descending artery, circumflex artery, right coronary artery involvement at the same time, the diameter stenosis greater than $50 \%$, or the degree of stenosis is greater than $50 \%$; left main plus any one of the vessels, the diameter stenosis greater than $50 \%$.

\subsection{Specimen Collection and Testing Methods}

Specimen collection: Before coronary angiography, early morning fasting venous blood $5 \mathrm{ml}, 1000 \mathrm{r} / \mathrm{min}$ Centrifuge $5 \mathrm{~min}$, collected serum, stored in $-70^{\circ} \mathrm{C}$ refrigerator, to be measured. Detection methods: The enzyme-linked immunosorbent assay (ELISA) quantitative determination of RBP4, LP-PLA2 concentration levels, kit provided by Wuhan Huamei Biotechnology Co., Ltd., microplate reader provided by Beijing Tianshi Technology Co.

\subsection{Statistical Methods}

Statistical analysis was performed with SPSS 16.0 software. Paired $t$ test was used to measure the data, and $\chi 2$ test was used for the count data. The difference was statistically significant $(\mathrm{P}<0.05), \mathrm{P}<0.01$ was significant difference $(\chi \mathrm{X} \pm \mathrm{S})$ and had statistical significance.

\section{Results}

\subsection{The Comparison of Concentration Between Groups of RBP4 and LP-PLA2}

The comparison of concentration among four groups of serum retinol binding protein 4, lipoprotein-related phospholipase A2 are described on Table 1. RBP4 concentration in AMI group, UAP group was significantly higher in the SAP group and the control group was low, the difference was statistically significant $(\mathrm{P}<0.05)$, AMI group and UAP group was no significant difference between the statistical significance $(\mathrm{P}>0.05)$. There was no significant difference between the SAP group and the control group (P> $0.05)$. The LP-PLA2 concentration in the clinical type of coronary heart disease was significantly higher than that in the 
control group $(\mathrm{P}<0.01)$. There was significant difference between UAP group and SAP group $(\mathrm{P}<0.05)$. There was significant difference between AMI group and UAP group and SAP group $(\mathrm{P}<0.01)$.

Table 1. Four groups of serum retinol binding protein 4, lipoprotein-related phospholipase A2 concentration comparison $(x \pm S)$.

\begin{tabular}{|c|c|c|c|}
\hline Group & $\begin{array}{l}\mathrm{C} \\
\text { ase }\end{array}$ & RBP4 & LP-PLA2 \\
\hline Control Group & 48 & $14.17 \pm 2.81$ & $150.63 \pm 32.81$ \\
\hline Acute Myocardial infraction & 15 & $25.36 \pm 8.63 *$ & $483.38 \pm 54.71_{\triangle}^{\mathbf{\Delta}_{\Delta}}$ \\
\hline Unstable angina pectoris & 71 & $19.72 \pm 5.27 *$ & $432.04 \pm 49.68^{\mathbf{\Delta} \#}$ \\
\hline Stable angina pectoris & 26 & $16.63 \pm 4.53$ & $395.27 \pm 32.90^{\mathbf{\Delta}}$ \\
\hline
\end{tabular}

Denote: Compared with the control group and stable angina $\mathrm{p}<0.05$; compared with the control group $\boldsymbol{\Delta} \mathrm{p}<0.01$; unstable angina, stable angina $\mathrm{p}$ $<0.01$; compared with stable angina $\mathrm{p}<0.05$.

\subsection{The Comparison of Concentration Among Different Coronary Artery Disease Groups}

The comparison of concentration of serum RBP4, LP-PLA2 according to coronary angiography results, patients with different coronary artery disease and control group are described on Table 2. The levels of RBP4 and LP-PLA2 in the three groups were significantly higher than those in the control group ( $\mathrm{P}<0.05)$. There was no significant difference between the three groups $(\mathrm{P}>0.05)$. LP-PLA2 concentration difference was statistically significant $(\mathrm{P}<0.05)$.

Table 2. The comparison of concentration between different coronary artery disease and control group serum RBP4, LP-PLA2 ( $x \pm S)$.

\begin{tabular}{llll}
\hline \multicolumn{1}{c}{ Group } & \multicolumn{1}{c}{ Case } & \multicolumn{1}{c}{ RBP4 } & \multicolumn{1}{c}{ LP-PLA2 } \\
\hline Control & 48 & $15.83 \pm 7.85$ & $150.63 \pm 32.81$ \\
Single-vessel & 42 & $19.84 \pm 6.25^{*}$ & $367.52 \pm 45.54^{*}$ \\
Double-vessel & 47 & $23.26 \pm 5.61^{*}$ & $446.37 \pm 32.54^{*}$ \\
Triple-vessel & 23 & $25.76 \pm 6.73^{*}$ & $483.75 \pm 51.23^{*}$ ム \\
\hline
\end{tabular}

Denote: Compared with the control group $* \mathrm{p}<0.05$; compared with single-vessel disease group \# $\mathrm{p}<0.05$; and double-vessel disease group $\boldsymbol{\Delta} \mathrm{p}$ $<0.05$.

\section{Discussion}

Abnormal lipid metabolism and inflammatory response is the main risk factor of coronary atherosclerosis and one of the trigger factors. RBP4 as a vitamin A carrier protein, is a newly discovered fat cell factor, the relative molecular mass of $21 \mathrm{KU}$. Numbers of animal and human studies have shown that when its level rising, which is closely related to the changes in insulin resistance, diabetes, central obesity, primary hypertension, lipid metabolism, such as changes in lipid profile and so on $[1,4]$, is a cardiovascular disease risk factors, may be directly or indirectly through a variety of ways involved in the occurrence and development of coronary heart disease, but the specific mechanism of action has not yet elucidated. May be associated with it is related to metabolic signals, inflammation and atherosclerosis of the link [5]. LP-PLA2, also known as platelet activating factor acetychydrocase (PAF-AH), contains 441 amino acids and has a relative molecular mass of 50,000 and is secreted by monocytes, macrophages, T lymphocytes and mast cells [6]. About $80 \%$ of LP-PLA2 in blood binds to LDL-C and 15-20\% binds to HDL-C [7]. LP-PLA2 hydrolyzes oxidized phospholipids derived from LDL-C into fatty acids, releasing both lysophosphatidylcholine and oxidized non-esterified fatty acids, both of which have significant proinflammatory effects and contribute to the formation of atheromatous plaques [7].

LP-PLA2 is an inflammatory marker associated with coronary artery disease. It is an independent risk predictor of coronary artery disease [8-9].

In the present study, we have shown that RBP4 concentration in AMI group and UAP group was significantly higher than that in SAP group and control group $(\mathrm{P}<0.05)$. There was no significant difference between each group $(\mathrm{P}>$ 0.05 ), indicating that coronary artery plaque block stability associated with RBP4 concentration, abnormal concentration may represent coronary plaque stability changes, leading to the instability of development, also may lead to coronary artery endothelial injury, plaque rupture, leading to acute thrombosis, that is, stable angina pectoris transferring to unstable angina pectoris, myocardial infarction development, or even sudden death. The RBP4 concentration in coronary artery disease group was significantly higher than that in the control group $(\mathrm{P}<0.05)$. There was no significant difference in the RBP4 concentration among the subgroups of CHD group $(\mathrm{P}>0.05)$. The results of this study is similar to the study of Liu and et al [10], indicating that RBP4 cannot reflect the degree and extent of coronary artery stenosis, but may become a risk stratification basis for further study.

The results showed that LP-PLA2 concentration in coronary heart disease was significantly higher than that in control group $(\mathrm{P}<0.01)$, indicating that LP-PLA2 may be a new inflammation marker associated with coronary heart disease, and may be directly involved in atherosclerosis and independently predict coronary event risk [11-12]. There were significant differences among the subgroups, and the concentration of LP-PLA2 in the two groups was significantly higher than that in the control group and the three-vessel disease group $(\mathrm{P}<0.05)$. The concentration of LP-PLA2 in the two groups was significantly higher than that in the control group ( $\mathrm{P}<0.01)$. There was a significant difference between the groups $(\mathrm{P}<0.05)$, indicating that LP-PLA2 could be used in the patients with severe coronary heart disease (CHD), the difference was statistically significant $(P<0.05)$, and the difference was statistically significant to a certain extent, can reflect the extent of coronary lesions or stenosis [13]. The study of Pan and et al [14] have shown that the results also confirmed that plasma LP-PLA2 levels can be used to indirectly assess the scope of coronary lesions. LP-PLA2 has strongly expressed within ruptured plaques with relatively weak staining in less advanced lesions [15].

\section{Conclusion}

In summary, the level of RBP4 is associated with the progression and progression of coronary artery disease, and do 
not correlate with the severity of coronary artery disease. The level of LP-PLA2 in patients with coronary heart disease increases with the progression and severity of coronary lesion. So according to the changes in LP-PLA2 levels can determine the deterioration of coronary heart disease, progress and degree of severity of coronary artery disease. Due to the small sample size of this study, the future's study still need to increase the sample size, more clinical centers to participate in further studies to confirm the value from the response to the clinical phenotype of coronary heart disease and coronary lesions.

\section{Acknowledgements}

The authors gratefully acknowledge support by the Huaibei People's Hospital for this work.

\section{References}

[1] Guo Jifang, Kong Fanhe. Retinol binding protein 4 and coronary heart disease correlation. Mudanjiang Medical College, 2013, 34: 12-14.

[2] Maiolino G, Pedon L, Cesari M, et al. Lipoprotein-associated phospholipase A2 activity predicts cardiovascular events in high rick coronary artery disease patients. PLOS One, 2012, 7(10): e48171.

[3] Han Yaling. Chang'an International Cardiovascular Forum. Chinese percutaneous coronary intervention guidelines. Chinese Journal of Cardiology, 2012, 40 (4): 252-254.

[4] Li F, Xia K, Sheikh MS, et al. Retinol binding protein 4 promotes hyperinsulinism-induced proliferation of rat aortic smooth muscle cells, Mol Med Rep, 2014, 9 (5): 1634-1640.

[5] Ge Ling, Cheng Xunmin, YANG Song, et al. Retinol binding protein 4 and lipoprotein-associated phospholipase A2 levels and coronary heart disease and coronary artery disease characteristics of the correlation analysis. Bengbu Medical College, 40 (8): 1102-1104.
[6] Fortunato J, Bláha V, Bis J, et al. Lipoprotein-associated phospholipase A2 mass level is increased in elderly subjects with type 2 diabetes mellitus. J Diabetes Res, 2014, 2014: 278063.

[7] Caslake M J, Packard C J. Lipoprotein -associated phospholipase A2 (platelet-activating factoracetylhydrolase) and cardiovascular disease. Curr Opin Lipidol, 2003, 14 (4): 347-352.

[8] Zhao Yong, Guo Zhibin. The value of lipoprotein-associated phospholipase A2 in predicting the risk of coronary heart disease. Journal of Applied Clinical Medicine, 2014, 15 (12): $1-3$.

[9] Ferguson J F, Hinkle CC, Mehta NN, et al. Translational studies of lipoprotein-associated phospholipase A2 in inflammation and atherosclerosis. J AM Coll Cardiol, 2012, 59 (8): 764-772.

[10] Liu Hai-liang, Li Guo-qing. Relationship between serum retinol-binding protein-4 and high-sensitivity C-reactive protein in patients with coronary heart disease. Journal of Clinical Internal Medicine, 2011, 28 (2): 92-94.

[11] Liu Xingjia, Zheng Xing, Qin Yongwen, et al. Lipoprotein-related phospholipase A2 activity in response to coronary angiography sclerosis degree, Journal of the Second Military Medical University, 2006, 27: 391-395.

[12] Cai A, Li G, Chen J, et al. Increased serum level of LP-PLA2 is independentiy associatated with the severity of coronary artery diseases: a cross-sectional study of Chinese population. BMC Cardiovasc Disord, 2015, 15 (1): 14.

[13] Wang Lili, Lei Changcheng. Lipoprotein-related phospholipase A2 and coronary heart disease related research progress. Modern Medicine and Health, 2015, 31 (1): 57-60.

[14] Pan Chen-liang, Peng Yu, Hu Xue-ting, et al. Correlation between lipoprotein-associated phospholipase A2 and acute syndromes. Clinical Cardiovascular Diseases, 2014, 30 (11): 962-965.

[15] Kolodgie F D, Burke A P, Skorija K S, et al. Lipoprotein associated phospholipase A2 protein expression in the natural progression of human coronary atherosclerosis. Arterioscler Thromb Vasc Biol, 2006, 26: 2523-2529. 